\title{
Tobacco use during adolescence may predict smoking during adulthood: simulation-based research
}

\author{
Raúl A. Borracci, M.D. ${ }^{a}$ and Andrés H. Mulassi, M.D. ${ }^{b}$
}

\begin{abstract}
Introduction. There is little information about the age of onset of smoking among adolescents and its continuation into adulthood. The objective of this study was to assess the influence of tobacco use during adolescence to predict the prevalence of adult smoking using simulation models.

Material and Methods. Five models were examined based on initiation and tobacco use rates among 421 adolescents. After simulating different scenarios, expected adult tobacco use rates were obtained and compared to those observed in a validation sample made up of 1218 adults.

Results. Models adequately predicted adult smoking rates by comparing them to data obtained using the validation sample (Markov: $37.6 \%$ versus $34.5 \%, p=0.109$; dynamic simulation: $32.0 \%$ versus $34.5 \%, p=0.197)$. The simulation demonstrated that smoking, at least, one cigarette per month during adolescence sufficed to predict adult tobacco use rates. Eliminating tobacco use during adolescence may reduce the rate of tobacco use among adults by $12.2-16.2 \%$.

Conclusions.Adolescent tobacco use models adequately predicted the proportion of smokers among adults. Scenarios of restriction regarding the age of onset of tobacco use showed the expected reductions in the rates of tobacco use among adults. Although it was not evaluated in this study, restricting tobacco use among adolescents may help to protect their health and would probably have an impact on the reduction of tobacco-associated mortality among adults. Key words:adolescents, smoking,simulation.
\end{abstract}

of Biomedical

Sciences,

Universidad Austral.

b. Council of

Cardiovascular

Epidemiology

and Prevention,

Argentine Society of Cardiology.

Buenos Aires,

Argentina.

E-mail Address:

Raúl A. Borracci,

M.D.:raborracci@gmail. com

Conflict of Interest:

None.

Received: 7-12-2014

Accepted: 10-2-2014 http://dx.doi.org/10.5546/aap.2015.eng.106

\section{INTRODUCTION}

Smoking is a preventable risk factor associated with multiple diseases worldwide. Particularly, Argentina appears to be in the advanced stage IV of the tobacco use epidemiological transition, ${ }^{1}$ with a smoking-related mortality of $16 \%$ out of all deaths and a prevalence of $33.4 \%$ between 18 and 64 years old. ${ }^{2}$

Smoking usually starts during adolescence, when youth first come into contact with cigarettes. ${ }^{3}$
Approximately $52 \%$ of adults refer to have started smoking between 12 and 17 years old, while 30\% started between 18 and 20 years old. ${ }^{3}$ In addition, recent studies have analyzed the influence of friendship social networks on smoking take-up among adolescents. . $^{4-6}$

Some of the measures proposed to reduce smoking prevalence among adults include reducing the initial contact with cigarettes during adolescence. However, simulation-based studies suggest that, for example, raising the legal age to buy cigarettes does not appear to have a significant impact on the reduction of smoking rates. ${ }^{7}$ In addition, reducing contact with cigarettes during adolescence might only have a late effect on the population's health and, in the worstcase scenario, it might just delay the take-up of smoking. ${ }^{8}$ Ferrante, D., et al. ${ }^{9}$ analyzed the influence of different tobacco control policies implemented in Argentina using simulation models in SimSmoke ${ }^{\circledR}$. Besides its usefulness to establish health measures aimed at reducing tobacco use, the authors recognize that there is little information about the age at smoking initiation and initial contact rates among adolescents.

Based on a sub-analysis of three prior surveys on smoking ${ }^{3,4,10}$ including more than 400 adolescents and 1500 adults from the town of Lobos (province of Buenos Aires), the objective of this study was to analyze how adolescent smoking may influence on the expected prevalence of tobacco use during adulthood using simulation models. 


\section{MATERIAL AND METHODS}

The study was conducted in four stages: 1) population or sources of data for model implementation, 2) model development, 3) simulations and result analysis, and 4) model validation.

\section{Population}

In order to implement models, raw data from two registries previously published by authors were subjected to a sub-analysis. Both registries corresponded to studies on smoking prevalence among adolescents and adults from Greater Buenos Aires. ${ }^{3,10}$ So as to make the sample epidemiologically homogenous, data used were only those corresponding to the town of Lobos, which included 421 adolescent students ( $54.4 \%$ were males, their age was $14.3 \pm 1.06$ years old $[\text { mean } \pm \text { SD] })^{10}$ and 386 adults ( $45.3 \%$ were males, their age was $43.2 \pm 10.6$ years old [mean \pm SD] $).{ }^{3}$ Based on the analysis of both registries, the following data were collected: adolescent tobacco use rates and initiation age, cessation rates, relapse and quitting in the adult sample. This information was used to implement different models and determine, through simulations, the expected prevalence of adult tobacco use based on adolescent tobacco use dynamics. The different rates, parameters and equations included in the models are summarized in Table 1.

\section{Model development}

The state transition diagram shown in Figure 1 was developed and used to implement three models based on Markov processes and one dynamic simulation model with stochastic components. The decision to develop three Markov models was based on having tried minor modifications while shaping matrices, all compatible with the general diagram shown in Figure 1. The diagram describes the dynamic of smoking take-up during adolescence and the transfer of the different tobacco use rates into adulthood. Based on this basic model, the different matrices of Markov processes and the dynamic simulation were implemented. According to adolescent smoker rates, an attempt was made to determine the expected rate of adult smokers using different simulations. "Contact with cigarettes" is defined as a situation where adolescents came into contact with cigarettes (smoked), but did not take up the habit (become smokers). "Adolescent smokers" are defined as those who smoked, at least, one cigarette per month. The most important assumptions included in simulations were that all adolescent smokers turned into adult smokers and that there were no health policies or interventions in place that would influence on the decision on whether or not to smoke. Different scenarios were proposed to know, on one side, the expected prevalence

TABLE 1. Rates, parameters and equations used for developing models (95\% confidence intervals are shown between parentheses)

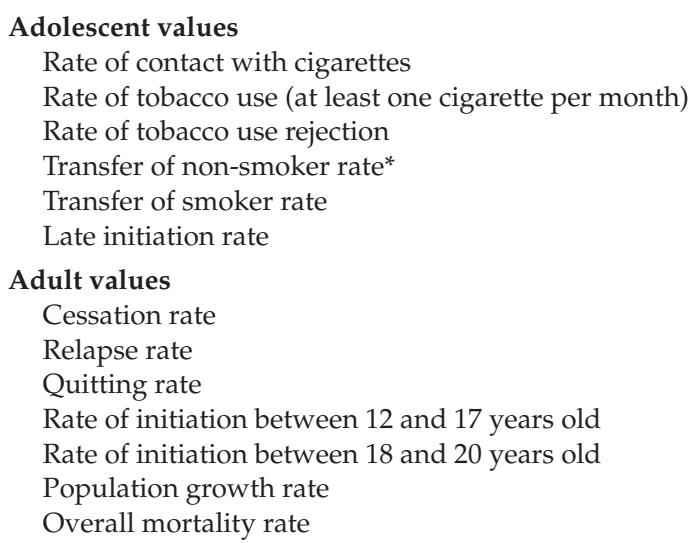

$$
\begin{gathered}
0.285(0.242-0.328) \\
0.467(0.419-0.515) \\
1 \text { - initiation rate } \\
1 \text { - (contact rate + late initiation rate) } \\
1.0 \\
1.477 \times \text { contact rate } x \text { initiation rate }
\end{gathered}
$$

$0.543(0.496-0.593)$

$0.552(0.502-0.602)$

$0.448(0.398-0.498)$

$0.523(0.473-0.573)$

$0.305(0.259-0.351)$

0.0101

0.0076

* The transfer of smoker and non-smoker rates assume what proportion of adolescent smokers become adult smokers and what proportion of adolescent non-smokers become adult smokers (values assigned are considered model assumptions). 
of adult smokers based on the smoking rates observed among adolescents and, on the other side, the expected prevalence after reducing the rate of contact with cigarettes during adolescence by $50 \%$ and $100 \%$.

\section{Data for model validation}

After performing simulations and obtaining the expected adult tobacco use rates, results were validated using the data obtained from the sub-analysis of two independent published registries..$^{4,10}$ This validation sample was made up of 1218 adults and included the results of a survey on tobacco use prevalence among the parents of previously surveyed adolescents ${ }^{10}$ and from an additional sample analyzed in the same town of Lobos. ${ }^{4}$

\section{Simulation and statistical analysis}

Several studies have recently highlighted that simulations are useful to test epidemiological models, including a comprehensive review in pediatrics. ${ }^{11,12} \mathrm{~A}$ stochastic process is a math concept that helps to represent a collection of random (stochastic) variables that progress based on another variable, generally over time. Each random variable in the process has its own probability distribution function and may or may not correlate to one another. Each variable or set of variables subjected to random effects or influences accounts for a stochastic process. ${ }^{13}$ For its part, a Markov process is a set of states and state transition probabilities where an initial state generates a series of successive and consecutive states using the product of matrices. Although Markov processes can easily handle feedback loops and point probabilities, a dynamic simulation performs better when using probability distributions. ${ }^{14}$

Based on the diagram shown in Figure 1, three Markov models were implemented according to the matrix product resolution technique into three Microsoft Excel $2003^{\circledR}$ spreadsheets. In addition, the dynamic simulation model was implemented into STELLA ${ }^{\circledR}$ Research 5.1.1 considering the probabilistic distribution (confidence intervals) of the different rates summarized in Table 1.

Prevalence data were expressed as percentages or proportions with the corresponding $95 \%$ confidence intervals (CIs). Continuous outcome measures were expressed as mean and standard deviation (SD), once the normality of distribution was proven using the KolmogorovSmirnov's goodness-of-fit test. For validation purposes, the adult sample size to compare the observed versus expected prevalences based on the models was established at $\mathrm{N}=1116$, with an 0.05 type I error, an 0.2 type II error $(80 \%$ power), an 0.35 prevalence, and an 0.08 minimum difference to be detected. In order to compare the predicted or expected data obtained through simulations to those observed in the validation sample, a null hypothesis of no difference between observed and expected values was

FIGURE 1. General diagram for simulation models

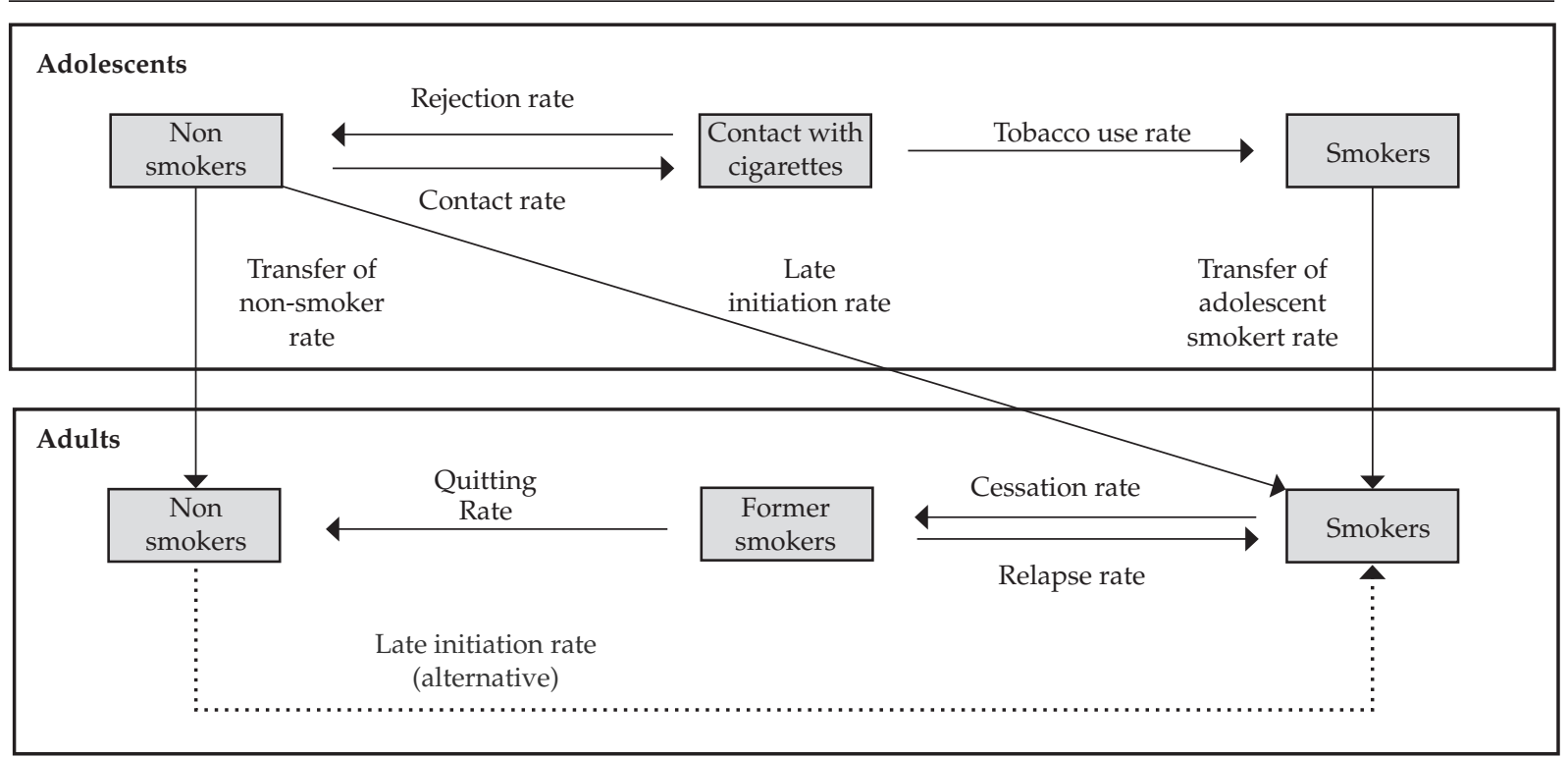


proposed; these estimates were also expressed as 95\% CIs. A chi-square test was used to compare each expected value obtained through simulations to the observed tobacco use prevalence in the validation sample. The level of significance was established at 0.05 and the SPSS $17.0^{\circledR}$ software was used to analyze data.

\section{RESULTS}

Figure 2 shows the results of simulations with the three models based on Markov processes. Each peak or trough level shows the expected proportion of adult smokers based on each model. Simulations predicted expected adult tobacco use rates of $29.5-37.6 \%$, according to the peaks reached in each Markov model. For its part, the dynamic simulation model predicted an adult tobacco use rate of $32.0 \%$ (95\% CI: 31.6-32.5\%).

Figure 3 shows the consequences of reducing contact with cigarettes during adolescence. Simulations demonstrated that eliminating all contact with cigarettes between 14 and 17 years old may reduce adult tobacco use rates by $19.7-31.5 \%$. In the validation sample of 1218 surveyed adults, the prevalence of tobacco use was $34.5 \%(n=420)(95 \%$ CI: $31.8-37.2 \%)$. Comparisons between the observed and predicted rates obtained from the different models are summarized in Table 2. Each model values correspond to, from left to right, expected adult

FIGURE 2. Results of Markov simulations and example of dynamic simulation showing the proportions of expected adult smokers according to each model
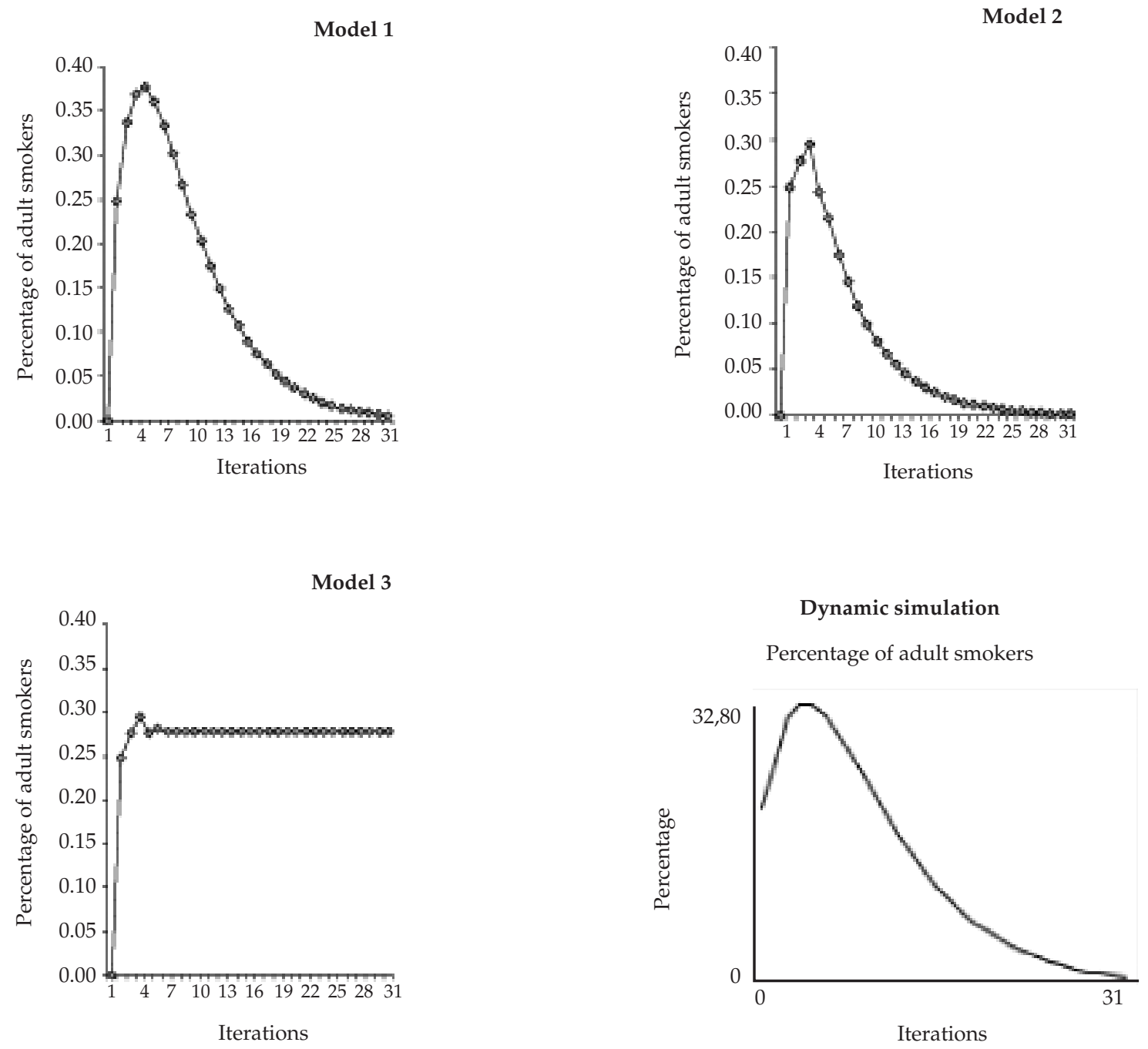
tobacco use rates based on the contact observed among adolescents (expected rate) and a 100\% and $50 \%$ reduction in contact with cigarettes during adolescence (RC100\%-50\%). Lastly, the RRR100\%$50 \%$ indicate the expected relative reduction in the rate of tobacco use following a $100 \%$ and $50 \%$ reduction in contact during adolescence. $P$ values correspond to the comparisons of each expected value obtained through simulations and the observed prevalence of tobacco use in the adult validation sample. Markov model 1 showed the best performance when compared to the validation sample (expected adult tobacco use rate: 37.6 versus observed rate: $34.5 \%, p=0.109$ ).
In addition, the dynamic simulation model also showed an adequate performance $(32.0 \%$ versus $34.5 \%, p=0.197)$. By using the simulation in two different scenarios for the $50 \%$ and $100 \%$ reduction of contact with cigarettes during adolescence, model 1 was observed to predict that reducing contact with cigarettes during adolescence to a half may decrease the rate of adult smokers by $7.2 \%$ (relative reduction) in the future, while completely eliminating contact with cigarettes (100\% reduction) may decrease the percentage of adult smokers by $16.2 \%$. In this latter case, the dynamic simulation model predicted a $12.2 \%$ relative reduction.

FIGURE 3. Markov simulations and example of dynamic simulation to analyze the effect of eliminating contact with cigarettes during adolescence on expected adult smoker rates
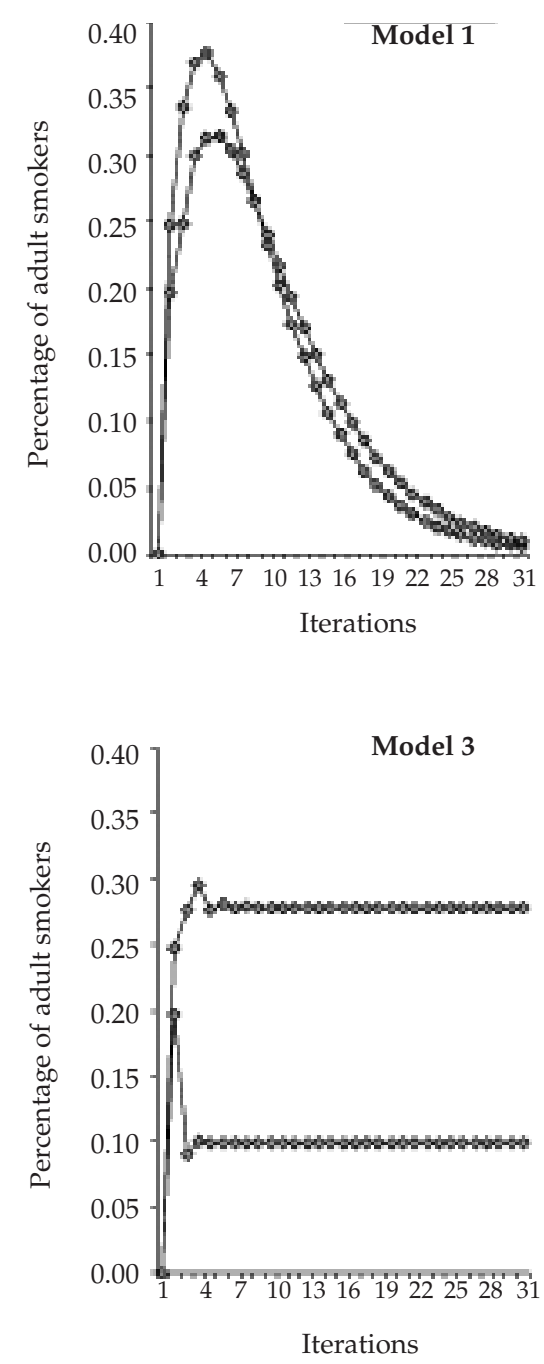

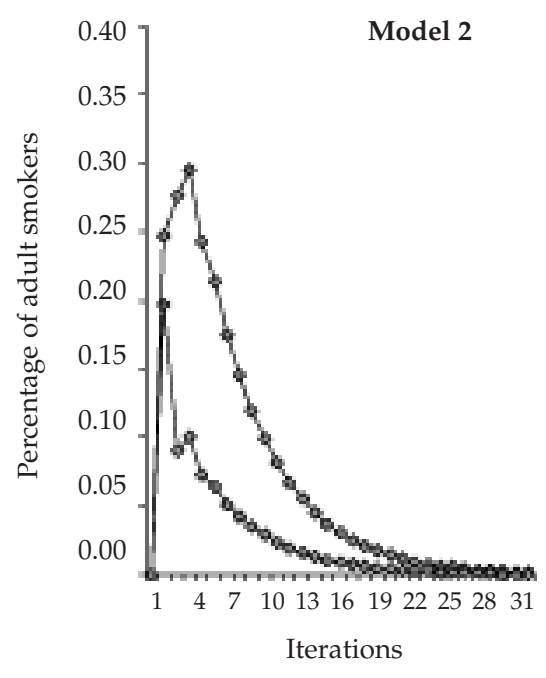

Dynamic simulation

Percentage of adult smokers

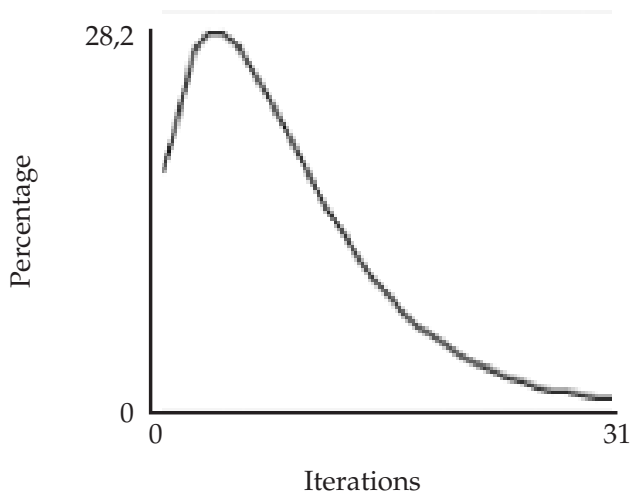




\section{DISCUSSION}

Models based on the dynamic of tobacco use among adolescents adequately predicted adult tobacco use rates when compared to the data observed in the validation sample. Model simulation indicated that smoking, at least, one cigarette per month during adolescence may predict future adult smoking rates. Particularly, Markov model 1 and the dynamic simulation performed best. A Markov-based simulation predicted adult tobacco use rates in a range from $34.9 \%$ to $40.3 \%$, while the prevalence observed ranged between $31.8 \%$ and $37.2 \%$. In addition, the dynamic simulation predicted lower rates and a shorter range, between $31.6 \%$ and $32.5 \%$. Almost all models indicated that reducing or eradicating an initial contact with cigarettes during adolescence may significantly decrease adult tobacco use rates since $52 \%$ of adult smokers referred to have started smoking during adolescence. However, the lack of contact in this period may only delay the decision to start smoking during adulthood and, therefore, an intervention aimed at adolescents would not have the desired effect. ${ }^{15}$ The models assessed in this study demonstrated that, in the worstcase scenario, eliminating contact with cigarettes during adolescence may reduce the adult smoker rate by $12.2-16.2 \%$ (dynamic simulation and Markov 1, respectively). If considering that interventions may eliminate smoking initiation in individuals younger than 18 years old and that none of them would ever smoke, the actual effect on smoking overall rates would still be discreet in the short term. It should be noted that the models only considered the most traditional independent factors related to smoking take-up, withdrawal and relapse, without any interventions. It should also be considered that, however, there are other factors associated with health intervention policies that may change smoking dynamics from adolescence into adulthood. Thus, smoking rates may vary as a result of prevention campaigns, smoke-free regulations, excise taxes, smoking cessation programs and raising the legal age to buy cigarettes. ${ }^{9}$ According to the general opinion, interventions aimed at reducing or eliminating contact with cigarettes during adolescence, such as raising the legal age, promoting a healthy lifestyle, providing industry incentives so that companies target their advertisements to groups who are legally capable of buying tobacco products or raise prices (probably the most effective intervention), are useful only if accompanied by deterrent policies also directed to adults. ${ }^{9}$ Although the consequences of smoking usually appear during adulthood, it should be noted that the reduction of tobacco use among adolescents has an educational impact on health and may lessen the possibility of contact with other addictive products. ${ }^{16}$

Undoubtedly, scenarios where contact with cigarettes among adolescents is restricted only embodies an ideal theoretical approach. In Argentina, the sale of cigarettes to minors under 18 years old is prohibited in order to reduce

TABLE 2. Expected adult tobacco use rates, obtained based on three Markov models and a dynamic simulation, according to tobacco use during adolescence

\begin{tabular}{lcccccc}
\hline & $\begin{array}{c}\text { Expected } \\
\text { rate }\end{array}$ & $\mathbf{p}$ & $\mathbf{R C ~ 1 0 0 \%}$ & $\mathbf{R C ~ 5 0 \%}$ & RRR 100\% & RRR 50\% \\
\hline $\begin{array}{l}\text { Markov model 1 } \\
\text { (95\% IC) }\end{array}$ & $37.6 \%$ & 0.109 & $31.5 \%$ & $34.9 \%$ & $16.2 \%$ & $7.2 \%$ \\
Markov model 2 & $(34.9-40.3)$ & & $(28.9-34.1)$ & $(32.2-37.6)$ & $(14.1-18.3)$ & $(5.7-8.7)$ \\
(95\% IC) & $29.5 \%$ & 0.008 & $19.7 \%$ & $22.2 \%$ & $33.2 \%$ & $24.7 \%$ \\
Markov model 3 & $(26.9-32.1)$ & & $(17.5-21.9)$ & $(19.9-24.5)$ & $(30.6-35.8)$ & $(22.3-27.1)$ \\
(95\% IC) & $29.5 \%$ & 0.008 & $19.7 \%$ & $22.2 \%$ & $33.2 \%$ & $24.7 \%$ \\
Dynamic simulation & $(26.9-32.1)$ & & $(17.5-21.9)$ & $(19.9-24.5)$ & $(30.6-35.8)$ & $(22.3-27.1)$ \\
(95\% IC) & $32.0 \%$ & 0.197 & $28.1 \%$ & $29.8 \%$ & $12.2 \%$ & $6.9 \%$ \\
\hline
\end{tabular}

Each model values correspond to, from left to right, expected adult tobacco use rates based on the contact observed among adolescents (expected rate) and a 100\% and 50\% reduction in contact with cigarettes during adolescence (RC100\%-50\%). Lastly, the RRR $100 \%-50 \%$ value indicates the expected relative reduction in the rate of tobacco use following a $100 \%$ and $50 \%$ reduction in contact during adolescence. All $\mathrm{p}$ values correspond to the comparisons between each expected value obtained with simulations and the tobacco use prevalence observed in the adult validation sample (34.5\%, 95\% CI: 31.8-37.2\%). 95\% CI: 95\% confidence interval. 
access to cigarettes during adolescence. However, almost $30 \%$ of surveyed adolescents had come into contact with cigarettes and $8 \%$ smoked on a daily basis.

Data required for model implementation was obtained exclusively from a single geographic area and population made up of both adolescents and adults. However, the adolescent sample included both rural and urban settings, which may be a study limitation. Even though working with a single population is an advantage for a homogenous design, it is clear that results cannot be extrapolated to the general population. Regardless of this, the smoking rate observed in the town of Lobos was $34.5 \%$, while the rate reported across Argentina according to the 2005 National Survey on Risk Factors was $33.4 \%{ }^{2}$

A limitation of the simulation used is that it was necessary to assume that all adolescent smokers would turn into adult smokers since there were no data available on how many adult non-smokers had smoked during adolescence. Another limitation is that, although the variation in smoking rates was studied across the different scenarios of reduction of initial contact with cigarettes, the time necessary to specifically reduce adult tobacco use rates was not taken into account since this was not a cohort study. Ahmad, S., et al. ${ }^{17}$ simulated the effect of raising the legal age to buy cigarettes in the USA to 21 years old over time, and concluded that such policy would reduce the prevalence of adult tobacco use rates only in the very long term. Anyway, with this intervention, the prevalence of adolescent tobacco use would immediately reduce to less than a half.

\section{CONCLUSIONS}

Markov model 1 and the dynamic simulation model, both based on adolescent tobacco use rates, adequately predicted the proportion of adult smokers. In addition, exploring different scenarios of age restriction for adolescent smoking initiation showed potential expected reductions in adult tobacco use rates. Although it was not examined in this study, restricting tobacco use among adolescents would help to protect their health, prevent the development of an addiction, and probably have an impact on the reduction of tobacco-associated mortality among adults.

\section{Acknowledgments}

To Daniel Ferrante, M.D., from the Ministry of Health of Argentina, for making a critical review of the manuscript.

\section{REFERENCES}

1. López AD, Collishaw NE, Piha T. A descriptive model of the cigarette epidemic in developed countries. Tob Control 1994;3(3):242-7.

2. Ferrante D, Virgolini M. Encuesta Nacional de Factores de Riesgo 2005: resultados principales. Prevalencia de factores de riesgo de enfermedades cardiovasculares en la Argentina. Rev Argent Cardiol 2007;75(1):20-9.

3. Área deInvestigación delaSAC,ConsejodeEpidemiologíay Prevención Cardiovascular de laSAC,Área del Interior dela SAC, Fundación Cardiológica Argentina. Prevalencia de los factores de riesgo coronario en una muestra de la población argentina. Estudio REDIFA (Relevamiento de los Distritos de la Sociedad Argentina de Cardiología de los factores de riesgo coronario). Rev Argent Cardiol 2002;70:300-11.

4. Mulassi AH, Borracci RA, CalderónJG, Vinay P, et al. Redes sociales de tabaquismo, consumo de alcohol y obesidad en adolescentes escolarizados de la ciudad de Lobos. ArchArgent Pediatr 2012;110(6):474-84.

5. Schaefer DR, Haas SA, Bishop NJ. A dynamic model of US adolescents' smoking and friendship networks. Am JPublic Health 2012;102(6):e12-8.

6. Schaefer DR, Adams J, Haas SA. Social networks and smoking: exploring the effects of peer influence and smoker popularity through simulations. Health Educ Behav 2013;40(1 Suppl):24S-32S.

7. Levy DT, Cummings KM, Hyland A. A simulation of the effects of youth initiation policies on overall cigarette use. Am J Public Health 2000;90(8):1311-4.

8. LevyDT,MabryPL,Graham AL, OrleansCT,etal. Exploringscenariostodramaticallyreducesmoking prevalence: a simulation model of the three-part cessation process. Am J Public Health 2010;100(7):1253-9.

9. Ferrante D, Levy D, Peruga A, Compton C, et al. The role of public policies in reducing smoking prevalence and deaths: the Argentina Tobacco Policy Simulation Model. Rev Panam Salud Publica 2007;21(1):37-49.

10. Mulassi AH, Hadid C, Borracci RA, Labruna MC, et al. Hábitos de alimentación, actividad física, tabaquismo y consumo de alcohol en adolescentes escolarizados de la provincia y el conurbano bonaerenses. Arch Argent Pediatr 2010;108(1):45-54.

11. ZubizarretaJR, ReinkeCE, KelzRR,SilberJH, etal.Matching for several sparse nominal variables in a case-control study of readmission following surgery. Am Stat 2011;65(4):229-38.

12. Cheng A, Auerbach M,HuntEA,Chang TP, etal. Designing andconductingsimulation-basedresearch.Pediatrics 2014;133(6):1091-101.

13. Hernández Sampieri R, Fernández-Collado C, Baptista Lucio P. Metodología de la Investigación. $4^{\text {ta }}$ ed. México DF: McGrawHill; Concepción o elección del diseño de investigación. 2006; Capítulo 7:157-233.

14. Borracci RA, MilinE. Modelos y simulación en investigación biomédica. Buenos Aires: Estudio Sigma; 2013. Págs.126.

15. Glantz SA. Preventing tobacco use - the youth access trap. Am J Public Health 1996;86(2):156-8.

16. Gielkens-Sijstermans CM,Mommers MA, Hoogenveen RT, Feenstra TL, etal.Reduction of smoking in Dutchadolescents over the past decade and its health gains: arepeatedcrosssectionalstudy.EurJPublicHealth 2010;20(2):146-50.

17. Ahmad S, Billimek J. Limiting youth access to tobacco: comparing the long-term health impacts of increasing cigarette excise taxes and raising the legal smoking age to 21 in the United States. Health Policy 2007;80(3):378-91. 